\title{
Lidil
}

Revue de linguistique et de didactique des langues

$57 \mid 2018$

Démarches créatives, détours artistiques et appropriation des langues

\section{Multilittératies et écriture avec des apprenants FLE}

Multiliteracies and Writing with FSL Learners

Noëlle Mathis et Muriel Cros

\section{OpenEdition}

\section{Journals}

Édition électronique

URL : http://journals.openedition.org/lidil/4877

DOI : $10.4000 /$ lidil. 4877

ISSN : 1960-6052

Éditeur

UGA Éditions/Université Grenoble Alpes

Édition imprimée

ISBN : 978-2-37747-048-8

ISSN : $1146-6480$

Référence électronique

Noëlle Mathis et Muriel Cros, « Multilittératies et écriture avec des apprenants FLE », Lidil [En ligne], 57 | 2018, mis en ligne le 01 mai 2018, consulté le 03 mai 2019. URL : http://journals.openedition.org/ lidil/4877 ; DOl : 10.4000/lidil.4877

Ce document a été généré automatiquement le 3 mai 2019.

(C) Lidil 


\title{
Multilittératies et écriture avec des apprenants FLE
}

\author{
Multiliteracies and Writing with FSL Learners
}

Noëlle Mathis et Muriel Cros

«J'attends toujours ces phrases assez rares, et elles
me choquent quand elles arrivent. Les phrases qui
arrivent en français. Aucune traduction
nécessaire. "
(EM, Caroline, P17)

\section{Introduction}

1 Cette étude se situe à l'Institut américain universitaire (IAU-ACM) d'Aix-en-Provence avec des étudiants de français langue étrangère (FLE) de niveau avancé. Lors de deux cours, ils sont exposés à des textes littéraires et à des œuvres d'art contemporain dans le but de mieux rencontrer les réalités nouvelles liées à la culture qu'ils découvrent, de composer des textes dits « de création» leur permettant de perfectionner la langue cible tout en développant des aptitudes à devenir auteur face au monde. Dans ces deux cours coexistant depuis trois ans, à raison de trois sessions par an, avec des groupes différents, nous avons constaté que la grande majorité des étudiants écrit des textes démontrant une appropriation des objets d'enseignement, mais déploie aussi une capacité surprenante (dans le sens où elle dépasse souvent nos attentes) à réfléchir et à manipuler la langue, tout en se positionnant de manière sensible. Nous avons alors questionné le dispositif didactique proposé, soit le recours, entre autres, à une littérature traditionnellement pensée comme difficile, voire inaccessible à un public non natif, combiné à des moments réflexifs réguliers d'autoanalyse. Nous nous sommes penchées sur ces derniers pour mieux comprendre notre action didactique. En effet, nous considérons les écrits des étudiants comme des réceptions du dispositif que nous mettons en place et supposons qu'en les analysant, nous pouvons mieux cerner le fonctionnement de nos cours. Nous avons alors procédé par allers-retours réguliers entre observations, méthodologie et 
théorie, pour éclairer les mécanismes propres au dispositif que nous tentons de décrire dans cet article. Ainsi, nous présentons d'abord le courant des multilittératies qui reflète nos pratiques, puis décrivons le contenu et la spécificité des deux cours et enfin procédons à l'analyse qualitative d'extraits de textes écrits par les étudiants.

\section{Les multilittératies et le corps}

2 L'étude s'inscrit dans le courant des multilittératies initié par le New London Group et représenté par Cope et Kalantzis (2000), dont nous ne proposons, faute d'espace, qu'un éclairage partiel. Ce courant insiste sur la dimension plurielle des contextes pédagogiques multilingues et multimodaux. En effet, ces auteurs conceptualisent les aspects qui sont à considérer lorsque des disciplines croisées telles que les nôtres (langues, littératures et arts) sont enseignées : la multiplicité des discours en circulation, les formes variées de représentation du langage et l'espace didactique qui les intègre en lien avec les identités des acteurs sociaux en présence. Le premier aspect considère la diversité des langues, qu'elles soient entre différentes zones géographiques et/ou contextes sociaux variés au sein d'une même langue (professionnelle, spécialisée, standard, littéraire, etc.). Le deuxième intègre les formes multimodales du langage combinant modes linguistiques, visuels, auditifs, gestuels et spatiaux avec les pratiques langagières et culturelles de communication. Selon Cope et Kalantzis, le terme multimodal est à comprendre comme l'intégration de ces différents modes dans les pratiques langagières, mais avec la notion d'un déplacement à travers ces modes selon des procédés de transcodage, soit l'opération de transformer une information fournie dans un code donné en une information équivalente dans un autre code ${ }^{1}$. Le troisième aspect concerne l'espace didactique selon une dimension critique incluant des pratiques situées et transformatives pour les apprenants comme les enseignants. En d'autres termes, l'approche des littératies multiples accorde un rôle essentiel à la multimodalité, soulignant l'accroissement et multiplicité des canaux de communication et l'importance des diversités culturelles et linguistiques dans le monde, mais également une conception du langage centrée sur la différence, le changement et la créativité. Les implications pédagogiques sont les suivantes: les formes de représentation du langage sont envisagées selon un processus dynamique plutôt qu'un processus de reproduction. Les apprenants sont considérés comme des acteurs sociaux en mesure de donner du sens à leurs expériences à travers leurs pratiques langagières. L'objectif de cette approche est de prendre en compte la sensibilité et la capacité créative des apprenants et de les ouvrir à la différence, au changement et à l'innovation.

3 Cette théorie sociale du langage comprend trois composants : le premier, available designs (les représentations comme ressources), reconnait les formes multiples de représentation du langage (notamment écrit). Il s'agit des ressources à disposition, des grammaires des divers systèmes sémiotiques (langues, images, illustrations, discours en circulation) à partir desquelles les pratiques discursives sont structurées. Le scripteur lit/écrit un texte en fonction de normes discursives qui changent d'un contexte à un autre, d'une institution à une autre, d'un groupe social à un autre, mais aussi d'une langue à une autre. Le deuxième, designing (l'acte de transformation des représentations), correspond à l'acte de reconfigurer et donner une nouvelle représentation à ces ressources. Dans cet acte, l'individu transforme ce qu'il connait et/ou ce qu'il se représente, selon une combinaison singulière liée à son expérience vécue. Il construit le sens grâce à un processus 
dynamique de transformation de signes en réponse à d'autres signes. Le troisième, redesigned, correspond à la transformation qui s'ensuit. L'acte de se signifier au monde donne à l'individu l'opportunité d'exprimer une subjectivité transformée et d'engager ses identités de manière créative. Ces trois composants nous servent à questionner les effets produits par le dispositif pédagogique proposé à des étudiants en cours de perfectionnement du français.

4 Toutefois, nous tenons à souligner le lien des littératies multiples avec le corps. Dans la suite de notre réflexion, pour envisager que créativité et transformation puissent se manifester dans nos cours, nous nous tournons vers les travaux de pédagogies critiques de Stein (2004). Stein considère le corps de l'apprenant comme étant au cœur du projet d'apprentissage, en valorisant ses multiples modes de représentation, ses expériences, langues et émotions. Le corps serait le site sensible de ressources multimodales et multisensorielles, un lieu qui donne sens à son environnement. Dans le cas d'écrits de locuteurs plurilingues, nous suggérons «que le corps soit le site multimodal, le locus réceptif de langues qu'il entend, qu'il ressent, qu'il voit écrites dans des scripts variés que ce soient des langues apprises et/ou des langues qui font partie de son environnement quotidien » (Mathis, 2013, p. 92-93). Les apprenants intègrent dans les textes qu'ils créent leurs sensations et leurs représentations de l'espace en fonction de la manière dont leurs langues peuvent advenir, être déplacées et mises en relation.

\section{L'art et la littérature en classe de langue}

5 Nous envisageons les textes littéraires et les œuvres d'art non pas comme finalités d'apprentissage, mais en ce qu'ils sont espaces de prédilection d'apparition et de réalisation du corps, tel que le souligne Aden (2008):

Les langues et les langages artistiques ont en commun de nous relier à nous-mêmes, aux autres et au monde au moyen de nos sens: apprendre à s'exprimer et à communiquer dans une langue, c'est d'abord développer sa capacité à entendre, voir et sentir. (p. 11)

6 Le recours à l'art et à la littérature convoque des expériences esthétiques et des confrontations à l'altérité qui contribuent à « une connaissance sensible et incorporée du monde » (ibid., p. 12). Plus spécifiquement, Godard (2015) indique que « la littérature offre comme deux "filtres" successifs sur la réalité : la singularité d'un regard et la singularité d'une lecture, dans leurs dimensions non pas seulement cognitive, mais affective, imaginaire et symbolique »(p.50). Mis en contact avec le texte littéraire, le lecteur «s'éprouve et se découvre » (Rouxel, 2015, p. 209), il « acquiert des savoirs expérientiels, de nature intuitive et empirique - l'aisthesis - qui le marquent bien davantage que les savoirs purement conceptuels » (ibid., p. 208).

7 Par ailleurs, Godard (2015) précise que «l'expérience littéraire de la langue enrichit non seulement notre connaissance et notre pratique de la langue, mais aussi notre capacité de penser la/les langues et notre relation à elle » (p. 271). Ceci se manifeste principalement à la fois dans le défi et le plaisir des multiples manipulations verbales qu'elle présente et autorise, dans la focalisation sur les dimensions concrètes et polyphoniques du signe et dans la pluralité des interprétations possibles des textes. En outre, à l'instar des enseignants s'inscrivant dans les approches interculturelles, le texte littéraire joue un rôle majeur «dans le développement d'une capacité à la fois de décentrement et de retour critique sur sa propre culture » (ibid., p. 49). En effet, comme le souligne Godard, 
«l'imaginaire hétérolingue qui se forge à la lecture des textes littéraires [...] affecte non seulement "la langue", mais aussi le sujet parlant : privé de son socle, il devient libre de se projeter dans des identités fluctuantes » (p. 283). Enfin, le passage d'une lecture à une écriture littéraire en L2 peut constituer pour l'apprenant un espace de liberté et d'expression créatrice qu'il n'aurait pas forcément en L1. Selon Dompmartin-Normand et Le Groignec (2015), le travail en atelier d'écriture littéraire avec un public FLE favorise des pratiques où «l'apprenant se découvre être-en-devenir, porteur d'une parole singulière qui l'émeut, le transporte, le transforme » (p. 67).

\section{Le dispositif didactique}

\subsection{Spécificités du dispositif didactique}

8 Le dispositif concerne deux cours universitaires conçus et enseignés par deux enseignantes qui, à travers cette étude, découvrent un positionnement didactique similaire. Chaque cours, de contenu spécifique, est suivi en moyenne par onze étudiants par semestre de treize semaines. Il s'agit donc de classes à petits effectifs. Les étudiants prennent l'un ou l'autre des cours, mais un tiers d'entre eux prennent les deux au cours d'un semestre donné. Globalement, ils sont âgés de 19 à 21 ans, viennent de colleges et universités divers des États-Unis. Un tiers de l'ensemble des étudiants des deux cours ont d'autres langues que l'anglais et le français dans leur répertoire verbal, issues de leur vie personnelle et familiale. Nous considérons les étudiants plurilingues car ils utilisent leurs langues quotidiennement. Durant un semestre d'études à Aix-en-Provence, ils perfectionnent la langue et la culture françaises, lors d'un programme study abroad ${ }^{2}$. Ils ont des spécialisations diverses et n'ont pas suivi de cours d'écriture créative et littéraire en français auparavant. À leur arrivée, leur compétence générale du FLE correspond à un niveau $\mathrm{B} 2$ selon le CECRL ${ }^{3}$.

Première spécificité : les continuums. Dans le dispositif, nous identifions les interrelations suivantes: entre la L1 et la L2, entre les pratiques orales et pratiques écrites, entre les écritures formelles de type analyse littéraire (textes standardisés) et informelles (écriture dite "de création", accompagnée de textes réflexifs), entre les activités en classe et à l'extérieur, entre les textes contextualisés et textes décontextualisés (textes sans référence aux contextes dans et grâce auxquels ils ont été écrits). Nous favorisons des espaces didactiques permettant aux apprenants de naviguer le long de ces continuums.

Deuxième spécificité : la réflexivitét et l'autoanalyse intégrées au processus didactique permettent, pour l'apprenant, un approfondissement de la partie d'exploration, de création et d'écriture. Les écrits réflexifs d'autoanalyse (désormais textes réflexifs) sont répartis sur l'ensemble du processus qui se présente non pas comme une boucle, mais comme une spirale ponctuée de moments d'analyse prolongeant l'intégration des éléments. Pour l'enseignant, le texte réflexif permet d'évaluer les étudiants avec des critères objectifs: proximité avec la proposition d'écriture, engagement, prise en compte des éléments proposés, niveau linguistique (grammaire, lexique, modalisation). Notons que ces activités de réflexion sont demandées par l'enseignant et comprises par l'apprenant comme le lieu de son évaluation, d'où également sa réponse cherchant à maximiser sa performance pour répondre aux attentes académiques. 
11 Troisième spécificité : la multimodalité ${ }^{5}$. Le dispositif combine multimodalité (matériel textuel et non textuel, données concrètes et réflexions abstraites), multidimensionnalité et pluralité dans les types d'exercices. À l'intérieur de ce cadre, l'étudiant fait ou non le lien entre les éléments. In fine, c'est parce qu'il doit exprimer la complexité des phénomènes observés hors de lui et en lui, que l'étudiant doit trouver les formes linguistiques nécessaires pour créer un texte singulier et donc parvenir à améliorer ses ressources linguistiques.

\section{Description des cours}

\subsection{Le séminaire "Traversées d'espaces dans des contextes interculturels »}

12 Il a pour but d'accompagner les étudiants dans leurs rencontres de la nouvelle culture. Il combine l'étude de textes littéraires, la découverte d'artistes, des expériences hors de la classe et la rédaction d'écrits créatifs dirigés. Comme le signale le tableau ${ }^{6}$ ci-dessous, le semestre suit les thèmes espace extérieur, corps/espace intérieur et relation aux autres.

Tableau 1. - Contenus du séminaire (3 heures hebdomadaires), printemps 2017.

\begin{tabular}{|c|c|c|c|}
\hline Thème & Littérature & Art & Expériences hors de la classe \\
\hline $\begin{array}{l}\text { 1) Relation avec } \\
\text { l'espace extérieur }\end{array}$ & $\begin{array}{l}\text { G. Perec } \\
\text { J. Roubaud } \\
\text { Oulipo }\end{array}$ & E. Pignon-Ernest & $\begin{array}{l}\text {-Réunion/discussion autour d'un } \\
\text { chocolat en ville. } \\
\text {-Exercice spatial (A. Boal) : la salle } \\
\text { de classe } \\
\text {-Visite en autonomie du musée } \\
\text { Granet } \\
\text {-Conférence sur la traduction }\end{array}$ \\
\hline $\begin{array}{l}\text { Synthèse } \\
\text { Individuelle }\end{array}$ & $\begin{array}{l}\text { Devoir créatif / } \\
\text { analytique } 1\end{array}$ & Créa1 & Semaine 5 \\
\hline $\begin{array}{l}\text { 2) Traduction de } \\
\text { l'espace intérieur } \\
\text { et corps }\end{array}$ & $\begin{array}{l}\text { H. Michaux } \\
\text { G. Luca }\end{array}$ & $\begin{array}{l}\text { Artistes- } \\
\text { photographes : } \\
\text { Delahaye, Ramette, } \\
\text { Li Wei, Darzack }\end{array}$ & $\begin{array}{l}\text {-Spectacle de danse } \\
\text { contemporaine (C. Rizzo) } \\
\text {-Coach vocal en classe (sur textes } \\
\text { d'H. Michaux) } \\
\text {-Danse dans les rues d'Aix (B. } \\
\text { Menaut) }\end{array}$ \\
\hline $\begin{array}{l}\text { Synthèse } \\
\text { Individuelle }\end{array}$ & $\begin{array}{l}\text { Devoir créatif / } \\
\text { analytique } 2\end{array}$ & Créa2 & Semaine 10 \\
\hline 3) L'autre & E. Guillevic & S. Calle & Conférence sur le plurilinguisme \\
\hline 4) La nature & & A. Goldsworthy & $\begin{array}{l}\text {-Visite parcours d'art de "Château } \\
\text { la Coste " } \\
\text {-Discussion départ (café en ville) }\end{array}$ \\
\hline $\begin{array}{l}\text { Synthèse } \\
\text { Individuelle }\end{array}$ & $\begin{array}{l}\text { Devoir créatif } 3 \text { / } \\
\text { performance }\end{array}$ & Créa3 & Semaine 12 \\
\hline
\end{tabular}

13 Chacun des constituants du séminaire (littérature, art et expériences hors de la classe) est mis en lien direct et explicite avec l'expérience de l'apprenant. Par exemple, une des propositions d'écriture est de «Marcher dans les rues d'Aix, s'asseoir dans un café [...]. S'inspirer très directement des “Travaux pratiques" d'Espèces d'espaces (Perec, 2000, p. 100-106). Choisir une de ses propositions et l'écrire ». Chaque constituant est considéré comme équivalent et complémentaire, l'objectif étant de les mettre en résonance. Ainsi, les interrogations sur l'histoire des espaces urbains dans les œuvres de Pignon-Ernest font écho à certains textes de Perec analysés en classe, qui font eux-mêmes écho à la visite en autonomie de musées aixois. De ce fait, les textes, les œuvres et les expériences 
se succèdent et apportent un angle d'approche différent tout en traitant du même thème. La progression s'effectue à la fois sur un axe vertical chronologique: les contenus du thème 1 mènent au thème 2 qui se prolonge dans le thème 3 , mais aussi sur un axe horizontal dans la mesure où les types de supports se répondent les uns aux autres à l'intérieur du même thème ou entre thèmes.

En termes d'objectifs pragmatiques, il s'agit de multiplier les utilisations orales et écrites de la langue cible ainsi que les pratiques créatives et analytiques adossées au vécu de l'apprenant. Ce dernier analyse ses propres expériences de vie par l'étude de textes et d'œuvres en relation avec l'espace extérieur, l'espace intérieur, ses ressentis nouveaux et les autres. Il développe sa pensée singulière et sa capacité à l'exprimer de mieux en mieux dans l'autre langue au fil du semestre. Ainsi, l'apprenant réalise un devoir créatif et analytique à la fin de chaque thème, soit trois « grands » devoirs synthétiques. Ce travail consiste à choisir parmi un ensemble d'expériences possibles (dans l'espace de la ville en thème 1 , dans l'espace de ses souvenirs et ressentis en thème 2 et dans l'espace de sa relation aux autres en thème 3 ). Les deux premiers devoirs doivent être scindés en deux parties disjointes : la partie créative (texte écrit avec une dimension expressive littéraire et/ou spatiale) et la partie analytique (texte réflexif sur l'expérience et le processus d'écriture). Le troisième et dernier écrit créatif est performé devant et avec le groupe.

\subsection{Le cours « Écrire en Provence - Littérature et culture régionale »}

Il a pour objectif l'émergence d'une écriture personnelle à partir de lectures d'auteurs provençaux ou ayant écrit sur la Provence. Le cours a été conçu pour que les activités d'écriture soient en lien avec le nouvel environnement des apprenants, selon trois thèmes: éloge de la Provence; rencontre de ses habitants et visiteurs; formes de résistance. Le tableau ci-dessous en montre les aspects principaux: neuf propositions d'écriture formulées à partir de l'étude de textes, de visionnage de films et d'un recueil final rassemblant des textes écrits pendant la session.

Tableau 2. - Contenus du cours (3 heures hebdomadaires), printemps 2017.

\begin{tabular}{|c|c|c|c|c|}
\hline Thème & Propositions d'écriture (I/E) ${ }^{1}$ & Forme-structure & Auteurs & Films \\
\hline 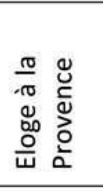 & $\begin{array}{l}\text { Souvenirs départ-arrivée (I) } \\
\text { Lumières \& petites choses (E) } \\
\text { Terrain des peintres (E) }\end{array}$ & $\begin{array}{l}\text { Fragments } \\
\text { Récit et zoom } \\
\text { cinématographique } \\
\text { Fragments poétiques }\end{array}$ & $\begin{array}{l}\text { G. Perec } \\
\text { J. Giono } \\
\text { J. De Romilly } \\
\text { A. du Bouchet }\end{array}$ & $\begin{array}{l}\text { Jean de } \\
\text { Florette } \\
\text { Manon } \\
\text { des sources }\end{array}$ \\
\hline & Texte réflexif \#1 & Ecriture analytique & & \\
\hline 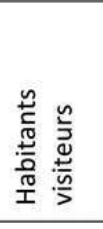 & $\begin{array}{l}\text { Marseille (E) } \\
\text { Le rêve du voyageur }(E) \\
\text { A une fontaine }(E) \\
\text { Lettre à un proche }(I)\end{array}$ & $\begin{array}{l}\text { Récit et métalepse } \\
\text { Monologue intérieur } \\
\text { Poème } \\
\text { Lettre }\end{array}$ & $\begin{array}{l}\text { A. Londres } \\
\text { M. Pagnol } \\
\text { J. Joyce } \\
\text { J. Sacré } \\
\text { J. Gardes } \\
\text { R. M. Rilke }\end{array}$ & $\begin{array}{l}\text { Marius } \\
\text { César }\end{array}$ \\
\hline & Texte réflexif \#2 & Ecriture analytique & & \\
\hline \multirow[t]{2}{*}{ 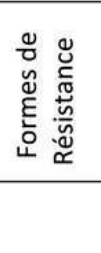 } & $\begin{array}{l}\text { Résistance : croyance (I) } \\
\text { Un être pas comme les autres } \\
\text { Recueil de textes collectif (I) }\end{array}$ & $\begin{array}{l}\text { Fragments et } \\
\text { anaphores } \\
\text { Libre (I) } \\
\text { Recueils imprimés }\end{array}$ & $\begin{array}{l}\text { R. Char } \\
\text { I. Huppert } \\
\text { J. Giono }\end{array}$ & $\begin{array}{l}\text { Un Hussard } \\
\text { sur les toits }\end{array}$ \\
\hline & $\begin{array}{l}\text { Analyse des processus } \\
\text { Café-lecture finale du recueil }\end{array}$ & Discussion en groupe & & \\
\hline
\end{tabular}



textes, d'explicitations de procédés d'écriture et d'une proposition concrète de partir de l'expérience sensible vécue. L'analyse des textes porte autant sur le sens (sentiment du narrateur, description d'un lieu, etc.) que sur les formes d'écriture (fragment, récit, monologue intérieur et lettre). Elle ouvre à des procédés d'écriture (effet de zoom, inscription du narrateur, métalepse et anaphore). Cependant, bien que ces formes d'écriture soient étudiées, il s'agit pour chacun de s'en inspirer sans la copier, ni l'imiter, en y inscrivant sa propre trace, dans l'imprévu et l'indéterminé. Deleuze (1993) rappelle qu'« écrire n'est certainement pas imposer une forme (d'expression) à une matière vécue [...], mais trouver la zone de voisinage » (p. 11). sensible comme matériau d'écriture. Les étudiants sont exposés aux villes d'Aix et Marseille, aux rues et marchés provençaux, aux fontaines, au terrain des peintres (lieu de Cézanne) de manière explicitement sensorielle. Les sorties sont l'occasion de manipuler un carnet d'écriture, de saisir bribes, impressions, couleurs, rugosité des surfaces, senteurs des herbes, mais aussi échos de langues diverses (celles des visiteurs, arabe aux marchés, toponymie provençale). Les films ouvrent à des discussions sur des éléments typiques de la Provence, dont les expressions et accents locaux.

18 Suite à chaque proposition, les étudiants écrivent chaque semaine, en classe ou chez eux, un texte dit «créatif» faisant l'objet d'évaluations formatives de trois types: la correction de la langue (grammaire, lexique, syntaxe) ; la cohérence et le style du texte ; la pertinence selon la proposition d'écriture. Tous les textes sont réécrits sans être notés. Ils sont également lus à haute voix pour recevoir les retours appréciatifs du groupe. En plus des textes créatifs, les étudiants écrivent un texte réflexif noté à la fin de chaque thème pour analyser leur processus de création : influence des auteurs, procédés et choix d'écriture, passerelles entre langues, sont questionnés.

\section{Méthodologie}

19 Notre corpus ${ }^{7}$ est composé des textes réflexifs produits par les étudiants au cours de deux semestres en 2016-2017 dans les deux cours présentés précédemment. Chaque étudiant en écrit environ dix pages, soit un corpus global de 400 pages dont nous utilisons des extraits non corrigés, pour un total de 44 étudiants. Nous considérons ces textes comme des traces repérables et analysables de leur processus de transformation. Dans ce corpus, nous avons repéré les extraits où ils ont fait référence aux auteurs ou artistes étudiés. Nous voulions vérifier qu'ils avaient fait un lien entre les textes littéraires et l'art proposés dans les cours et leur propre expérience. Nous avons constaté des mentions multiples aux auteurs sous diverses formes : citations, comparaisons (comme $\mathrm{X}$ ), reprise de la pensée de l'auteur (X parle de). De plus, ces mentions étaient accompagnées de la présence d'autres traces qui nous ont questionnées (marqueurs d'appropriation personnelle, adjectifs évaluatifs, références à la biographie, identifications ou mises à distance des auteurs). Nous avons tenté d'organiser ces observations afin de mieux cerner les effets du dispositif sur les apprenants. C'est alors que les trois composants décrits par les multilittératies nous ont semblé pertinents, soit les représentations comme ressources, l'acte de transformation des ressources, la transformation subjective. Nous avons donc entrepris un repérage systématique dans l'ensemble du corpus des extraits correspondant à notre compréhension de ces composants. Ces derniers sont souvent tissés les uns aux autres 
tout en étant discernables les uns des autres, certains restant dans des zones intermédiaires - cela semblait confirmer le caractère non linéaire et non déterministe d'un processus de création. Il n'est pas surprenant que la quasi-totalité des apprenants (dix sur onze par classe) aient produit des écrits qui relèvent du composant représentations comme ressources puisqu'ils répondaient à des devoirs universitaires dans chaque cours. Toutefois, il est plus surprenant qu'une forte proportion (sept sur onze en moyenne par classe) relève des deux autres composants. C'est ce dernier constat qui nous a incité à préciser les formes que prenaient ces trois composants pour nous aider à mieux cerner le fonctionnement du dispositif.

\section{Traces du processus dans les écrits}

Nous avons sélectionné des extraits d'écrits d'étudiants produits dans les deux cours 8 . Notre objectif ne vise pas l'exhaustivité : il est de montrer la pluralité et l'intrication des positionnements discursifs des étudiants dans les textes réflexifs.

\subsection{Représentations comme ressources}

Dans les extraits suivants, les étudiants se représentent, en les présentant, les ressources diverses proposées par les cours. Ils citent les auteurs, les artistes et les éléments de l'expérience en explicitant un lien personnel avec ces derniers. On isole les mentions aux auteurs et artistes, les identifications à ces derniers et la mise sur le même plan des contenus académiques et des expériences hors de la classe.

\subsection{Mention de l'auteur ou de l'artiste en tant que tel}

Il peut s'agir d'une simple mention à l'auteur, à l'ouvrage, avec citation complète avec ou sans guillemets, ou l'ensemble de ces options :

Dans le livre « Marcher ; Éloge des Chemins et de la Lenteur », David le Breton parle beaucoup de l'idée des gens dans une relation avec leur environnement et comment ils interagissent avec les lieux. Il introduit le concept que le marcheur «transforme la ville en un théâtre dont il occupe la première loge. » Quand j'ai réfléchi à cette citation et à ce que je veux faire, j'ai pensé de plus en plus à la fontaine qui se trouve sur la place des Quatre Dauphins. (SC1, Maxine, A16)

Maxine cite le titre du livre et son auteur, en mentionne la pensée ( $X$ parle de ») et propose une citation directe entre guillemets. Ensuite, elle transfère le résultat de sa réflexion («j'ai réfléchi », «j'ai pensé») au choix du lieu précis qu'elle décide d'explorer et à ce qu'elle « veu[t] faire ». L'explicitation de la ressource conceptuelle est mise en lien avec l'affirmation du choix personnel de l'action future.

Jean Giono m'a enseigné «Les vrais richesses » pour lesquelles je devrais chercher quand je visite à une nouvelle ville. (EA1, Cole, A16)

Cole cite Giono et le titre de l'œuvre pour l'enseignement qu'il en a tiré et qu'il généralise comme moyen de découvrir tout nouvel espace.

\subsection{Identification avec l'auteur}

L'étudiant s'identifie avec l'auteur et exprime son ressenti : 
Quand je suis arrivée ici, mon français était effrayant. J'ai eu peur de parler, je n'ai pas compris la culture ici en France, toujours bizarre pour moi ; Henri Michaux l'a dit mieux : «il faut un obstacle nouveau pour un savoir nouveau ». (SF, Rebecca, A16)

Rebecca explicite la difficulté de son expérience ("effrayant», "pas compris", «bizarre »): on remarque comment langue et culture sont étroitement liées par la proximité et la juxtaposition «j'ai eu peur de parler, je n'ai pas compris la culture ». Elle nomme et cite l'auteur entre guillemets qui «l'a dit mieux» pour exprimer sa propre pensée.

L'étudiant peut aussi partager une expérience particulière avec l'auteur :

Tous ces lieux étaient uniques et étaient aussi la source d'inspiration pour quelques auteurs que j'ai mentionné [...]. L'opportunité d'aller à un lieu qui a inspiré les textes que nous avons lu était incroyable et c'est grâce à cet opportunité que mon écriture est devenu plus réaliste. (EF, Berkley, P17)

Berkley pointe le caractère exceptionnel (« uniques », " incroyable ») de l'expérience proposée : "aller à un lieu qui a inspiré les textes». Elle marque une relation causale ( " c'est grâce à ») sur ce qu'elle perçoit de son écriture « devenu plus réaliste ». Le fait que les auteurs (ou artistes) aient écrit (ou peint) dans les lieux visités stimule l'acte d'écriture.

\subsection{La ville ou les expériences comme ressources}

L'environnement et les expériences qui y sont vécues servent de ressources pour le processus de création de la même façon que les contenus étudiés en classe :

La ville m'a permet de vagabonder avec un esprit ouvert en regardant les petits détails. C'est grâce à mon exploration de la ville que mon écriture est devenu plus précis et plus détaillé. (EF, Berkley, P17)

Berkley affirme un lien logique entre expérience concrète dans l'espace et style personnel d'écriture : la ville dans sa globalité est conçue comme source de façonnage de l'écriture. On remarque le choix du même mot " petits détails » lors de l'exploration répondant au style « plus détaillé » rendu possible par l'expérience.

La ville est devenue mon théâtre, un voyage artistique (comme Bernard Menaut) et

j'ai appris à tout recevoir même si je ne comprenais pas toujours, comme avec mon

expérience avec l'une des expositions au Château La Coste. (SF, Audrey, A16)

Tout en attestant l'évolution survenue (" devenue », "j'ai appris à recevoir »), Audrey se positionne en relation avec l'intervenant-artiste (comme X), ainsi qu'avec une activité obligatoire ("comme avec mon expérience avec l'une des expositions ») : elle trace un parallèle entre son expérience globale (« un voyage artistique»), sa faculté «à tout recevoir » même sans tout cerner et certaines expériences très précises proposées hors de la classe.

\subsection{Acte de transformation de ses représentations}

Dans les extraits suivants, les étudiants expriment la transformation en cours des ressources à disposition, que ce soit la recherche de voix en L2, la conscientisation de son positionnement ou l'explicitation de déplacements effectués dans le processus d'écriture.

\subsubsection{Recherche de voix dans une autre langue}

L'étudiant problématise les défis liés au passage de la L1 vers la L2 lors de l'écriture : 
Dans tous mes écrits, j'essaye de trouver la partie de moi qui est française. Mais, elle n'est pas toujours facile à trouver. [...] Il y a toujours cette question : est-ce que mon style d'écriture en anglais peut être traduit en français? Ou dois-je trouver un style complètement nouveau ? Est-ce que le style se traduit? [...] Quand les mots ou les phrases ne viennent pas à moi en français, je les écris en anglais. Principalement des mots et des expressions, mais de temps en temps, je dois jeter des phrases entières parce qu'elles refusent d'être français. (EA2, Caroline, P17)

Caroline questionne identité (" partie de moi qui est française ») et style d'écriture selon la langue utilisée («Est-ce que le style se traduit?») en explicitant son expérience de résistance linguistique («quand les mots ou les phrases ne viennent pas »). Le texte est jalonné de modalisateurs exprimant effort et difficulté («j'essaye de trouver», «pas facile », «ne viennent pas») et questions ("dois-je trouver un style nouveau?»). La personnification de phrases qui « refusent d'être français(es)» exprime le défi d'écrire en L2. L'importance réside dans les solutions pragmatiques qu'elle trouve et les stratégies qu'elle emploie : écrire en langue maternelle ou supprimer.

\subsubsection{Positionnement dans son processus de création}

\section{Il s'agit également de marquer sa différence par rapport à l'auteur :}

Dans une façon différente que Guillaume Apollinaire, j'ai joué avec le placement des mots pas pour un jeu intellectuel mais plutôt émotionnel. J'ai voulu que [...] la forme aide le lecteur/la lectrice dans sa propre découverte de son espace intérieur autant que le mien. (SC2, Janae, A16)

Janae explicite son projet d'écriture en se mettant à distance («dans une façon différente ») par rapport à l'auteur qu'elle nomme, dont elle évalue l'intention (« un jeu intellectuel ») et éclaircit la sienne (" plutôt émotionnel »). Elle pose trois entités : le « je » écrivant, l'auteur et le lecteur et justifie ses choix pour l'action voulue sur le lecteur: «que la forme aide [...] dans sa propre découverte». La relation entre le «je » et son « lecteur » surpasse sa relation initiale avec l'auteur, par une mise en équivalence («son espace intérieur autant que le mien »). On retient une intention claire et précise dans son processus de création.

\subsubsection{Explicitation du cheminement}

Les étapes suivies en vue du projet d'écriture sont expliquées :

Quand j'ai choisi la première option de m'asseoir dans un café et faire des observations, je n'ai pas jamais pensé que j'arriverais à faire ce projet. J'étais sûre que je ne pourrais pas trouver un thème ou un sujet pour réussir dans ce devoir. Mais, au moment que je m'est assis dans la chaise du café Carrefour Bar, et j'ai commencé à penser sur mon entourage, le chose le plus évident m'a donné l'idée pour mon projet : la pluie. (SC1, Rebecca, A16)

Rebecca décrit son cheminement en deux étapes : la première mentionne sa certitude de ne pouvoir réussir ("je n'ai pas jamais pensé », "j’étais sûre que je ne pourrais pas ») alors que la deuxième explicite le moment du revirement, grâce à l'opposition (« mais »), aux indices temporels (" au moment que», «j'ai commencé à penser») et enfin le déclencheur qui «a donné l'idée »: la pluie. C'est l'inscription corporelle concrète dans un espace précis qui livre la clé de faisabilité.

Le procédé d'écriture est identifié :

J'ai trouvé que j'utilise la notion de zoom comme Giono. (EM, Eli P17)

Eli explicite la similarité de sa stratégie avec celle de l'auteur étudié. 


\subsection{Transformation}

31 Certains écrits, surtout en fin de semestre, attestent de la perception d'une subjectivité transformée : nouvelle perception de soi et/ou de l'acte d'écriture.

\subsubsection{Nouvelle perception de soi}

L'étudiant explicite comment le projet de création l'a transformé :

Finalement, à cause de ce projet, j'ai gagné une nouvelle gratitude pour moi-même, aussi, car j'ai réalisé que j'ai la capacité d'explorer quelques grandes pensées philosophiques, dans une langue étrangère, et de résumer tout ce que je me suis sentie dans une forme créative. Ce projet m'a vraiment surpris et je sais que je garderai ces leçons pour toujours. (SC1, Berkley, P17)

Berkley exprime de façon positive le fait d'avoir surmonté la difficulté objective du travail d'écriture. Cela aboutit à un sentiment de revalorisation personnelle («j'ai gagné une nouvelle gratitude »), de conscience de ses capacités («j'ai réalisé que j'ai la capacité »), d'être en mesure de créer ("dans une forme créative») et de satisfaction ouvrant sur l'avenir (« je garderai ces leçons »).

\subsubsection{Nouvelle perception de l'acte d'écriture}

L'écriture est envisagée d'une nouvelle manière :

Je suis habituée maintenant à l'inconnu. Je recommence mon niveau de l'écriture en écrivant dans une nouvelle langue [...] De plus, j'ai appris comment à écrire comme les auteurs renommé à Aix-en-Provence [...] Sacré, un auteur si capable d'observer sans jugement ou René Char, un poète de la résistance, grâce auxquels, au fur et à mesure, mon écriture français devient plus libres et alignés avec ce que j'ai envie de dire. (EF, Sophia, P17)

Sophia valide un changement accompli («je suis habituée maintenant », « sont devenus », " au fur et à mesure ») : depuis « l'inconnu » jusqu'au sentiment de liberté ressentie aussi bien dans sa faculté d'utiliser L2 que dans sa faculté d'exprimer ce qu'elle désire. L'articulation du passé-composé/présent renforce le marqueur de l'accompli. De plus, la mention des auteurs étudiés et leurs attributs précise la transformation perçue.

\subsection{Corps-émotions}

La référence au corps semble fondamentale :

Les cinq sens ont joué un grand rôle et j'avais l'impression que j'étais capable de vraiment voir le monde tout autour de moi. La sensation de la chaleur du soleil sur ma peau pendant que j'étais assis sur le sol de rochers - c'était en ce moment-là où je me suis jamais senti avec l'extérieur et avec moi-même. Pour cette raison, mon écriture est devenue beaucoup plus puissante. (EM, Israel, P17)

Israel explicite le recours à la sensorialité («les cinq sens») qui lui a permis d'être présent (« voir le monde tout autour de moi »). Il exemplifie en évoquant « la sensation de chaleur» ayant contribué à l'expérience du ici-et-maintenant, et résultant en une écriture plus riche («mon écriture [...] plus puissante »).

Le désir d'exprimer ses sentiments passe par le corps :

Pour décrire vraiment mes sentiments, j'ai essayé d'utiliser le mots avec des sons plus mélodieux pour refléter mon état de joie. (SC2, Maxine, A16) 
J'ai aussi comme la sensation d'être bouleversé par la nature, au centre-ville et aussi au Terrain des peintres. (EA1, Ben, P17)

La nécessité d'écrire permet de mettre à jour ses émotions :

Ce projet m'encourage à continuer à écrire après que je retourne chez moi parce que c'est un bon exercice pour la santé émotionnelle et j'ai aimé mon exploration de la poésie pendant mon temps ici. (SC2, Michelle, A16)

\subsection{Discussion}

Cette étude fait émerger des réflexions du point du vue des identités des apprenants : bien qu'ils écrivent en français langue cible, bien que les extraits choisis ne montrent aucune trace explicite d'autres langues, ils (se) réfléchissent, questionnent leurs choix et, ce que nous nommerions, leur être-de-langues. On a l'impression qu'ils écrivent en français comme s'il s'agissait non plus d'une langue à apprendre, mais d'une langue à incorporer, dans la rencontre avec auteurs, artistes, lieux, à travers l'expérience et l'environnement propice à la création. Incorporer la langue se fait dans l'acte de créer et de ressentir, qui lui-même émerge de la difficulté de l'épreuve : l'écriture et la création représentent un défi dont il faut trouver la clé. Ils acceptent - à un certain moment - de ne pas toujours comprendre et de ne pas forcément trouver réponses à leurs questions. Ils conçoivent que le passage d'une langue à l'autre n'est pas de l'ordre de la traduction, qu'il faut attendre avec patience que la langue se présente, que ses mots à soi adviennent avec la pratique pour qu'ils deviennent leur langue, que la recherche n'est pas celle dans un dictionnaire, mais celle de l'écoute d'un espace intérieur propre à chacun, que la langue devient objet de création en réponse à des sensations et que l'inconnu fait partie de cette démarche. En bref, la sollicitation du corps-émotions se situerait au cœur du processus, du désir de créer, dans cette fabrication d'un nouvel être-au-monde que semble engendrer le dispositif. En d'autres termes, la langue, l'appropriation des langues, répondraient à une incorporation (des grammaires, lexiques, sons, etc.) propre à la sensibilité de chacun dans un contexte culturel et didactique plurilingue donné.

Par ailleurs, les écrits réflexifs semblent fonctionner comme système de validation des appropriations langagières et confirmation des déplacements identitaires engendrés : le « dire » correspondrait au «faire». De plus, il semblerait bien y avoir continuums entre les éléments de contenus étudiés dans les cours qui fonctionnent comme des points entre lesquels les apprenants font (ou pas) les liens nécessaires à l'advenue du «sens », c'est-àdire à la compréhension des textes, artistes et expériences ainsi que l'aptitude à l'exprimer en L2. L'approche multimodale favoriserait ce travail de mise en relation et en cohérence des éléments par l'apprenant. C'est dans le processus de cette mise en mots qu'il réussit à s'approprier la langue étrangère tout en trouvant sa langue dans la langue. La principale caractéristique de ce dispositif est sans doute son horizontalité, correspondant à l'absence de hiérarchisation entre les constituants (littérature, arts, expériences hors de la classe). En outre, les deux cours sont mis en situation de complémentarité : partageant de nombreux positionnements, ils sont mis en résonance dans l'expérience didactique de l'apprenant. 


\section{BIBLIOGRAPHIE}

ADEN, Joëlle. (2008). Apprentissage des langues et pratiques artistiques. Paris : Éditions Le Manuscrit.

CoPE, Bill \& KALANTZIS, Mary. (2000). Multiliteracies: Literacy Learning and the Design of Social Futures. New York : Routledge.

Dompmartin-Normand, Chantal \& Le Groignec, Anne. (2015). Un atelier d'écriture créative en FLE : tissages et apprentissages. Éducation et sociétés plurilingues, 38, 59-71.

Deleuze, Gilles. (1993). Critique et clinique. Paris : Les éditions de Minuit.

GODARD, Anne. (2015). La Littérature dans l'enseignement du FLE. Paris : Éditions Didier.

MARQUILLÓ LARRUY, Martine. (2012). Littératie et multimodalité ici \& là-bas. Les cahiers de l'ACEDLE, 9(2), 47-84. Disponible en ligne sur <https://acedle.org/old/IMG/pdf/03_Marquillo-Larruy.pdf>.

MATHIS, Noëlle. (2013). Identités plurilingues et création textuelle en français langue étrangère : une approche sociolinguistique d'ateliers d'écriture plurielle (Thèse de doctorat, Université d'Avignon). Disponible en ligne sur <www.theses.fr/2013AVIG1121>.

MolinIÉ, Muriel (dir.). (2006). Biographie langagière et apprentissage plurilingue, Le français dans le monde. Recherches et applications, 39.

PEREC, Georges. (2000). Espèces d'espaces. Paris : Éditions Galilée.

RouXEL, Annie. (2015). De l'importance de l'expérience esthétique dans la formation du lecteur. Dans J. Van Beveren (dir.), Littérature, langue et didactique (p. 205-219). Namur : Presses universitaires de Namur.

STEIN, Pippa. (2004). Representation, Rights, and Resources: Multimodal Pedagogies in the Language and Literacy Classroom. Dans B. Norton \& K. Toohey (eds), Critical Pedagogies and Language Learning (p. 95-115). Cambridge, UK : Cambridge University Press.

\section{NOTES}

1. Pour éclairer cette notion, on pensera à une activité proposée dans le cours d'écriture qui consiste à se rendre au Terrain des peintres (lieu où Cézanne a peint la Sainte-Victoire près de 80 fois) et à écrire in situ, selon son propre ressenti, sous forme de fragments, ce que le peintre a peint.

2. Aux États-Unis, il est d'usage qu'un étudiant fasse un semestre d'études à l'étranger durant son Bachelor. Les crédits obtenus dans l'université étrangère sont transférés dans l'université d'origine.

3. Cadre européen commun de référence des langues.

4. Molinié (2006) précise que les activités réflexives et leur mise en mots contribuent à l'apprentissage des langues.

5. Cf. Marquilló-Larruy (2012) entre autres.

6. Les composantes étant des dynamiques dans le processus de création, les éléments présentés dans le tableau des deux cours montrent un parcours évolutif complexe, mais sont réducteurs, 
car d'autres activités écrites et orales telles qu'entrées de journaux, discussions en classe et analyses stylistiques n'y figurent pas.

7. Code corpus: S-Séminaire sur l'espace. E-Cours d'écriture. P17-Printemps 2017. A16Automne 2016. C1(2)-Premier (deuxième) devoir créatif/analytique dans le séminaire. A1(2)Premier (deuxième) texte d'analyse. M-Examen de mi-parcours. F-Examen final.

8. Soit un échantillon de 12 étudiants sur 44, 7 ayant pris les deux cours et 5 l'un des deux.

\section{RÉSUMÉS}

Cette étude examine un dispositif didactique combinant des textes littéraires et des œuvres d'art contemporain à partir de l'analyse qualitative des productions écrites d'apprenants FLE. Nous expliquons en premier lieu le courant des multilittératies dans lequel elle s'inscrit, notamment à travers une théorie sociale du langage se déclinant en trois composants : les représentations comme ressources à disposition, le processus de transformation de ces ressources, la subjectivité transformée qui en découle. Puis nous décrivons les principaux éléments qui constituent ce dispositif et analysons des écrits autoréflexifs d'apprenants à travers la déclinaison de ces trois composants. Comment explicitent-ils leurs processus d'écriture et d'appropriation des contenus ? Comment la multimodalité, l'horizontalité et la place attribuée au corps constituentelles des éléments déterminants dans ces processus ? Quels enseignements peut-on en tirer pour nos pratiques pédagogiques?

This study examines an educational program combining literary texts and contemporary works of art based on qualitative analysis of the written productions of FSL learners. We begin by explaining the pedagogy of multiliteracies in which it is embedded, notably through a social theory of language divided into three components: representations as available resources, the process of transforming these resources, and the transformed subjectivity that results from them. Then we propose an analysis of the self-reflective writings of learners through the declination of these three components. How do they explain their processes of writing and appropriation of content? How do multimodality, horizontality and body constitute decisive elements in these processes? What lessons can we draw from this for our pedagogical practices?

\section{INDEX}

Keywords : multiliteracy, FSL, writing, literature, body

Mots-clés : multilittératies, FLE, écriture, littérature, corps

\section{AUTEURS}

\section{NOËLLE MATHIS}

LIDILEM, Université Grenoble Alpes

\section{MURIEL CROS}

IAU-ACM, Aix-en-Provence 